\title{
Yo, Contigo. El Programa de Mediación entre Iguales, desde la Perspectiva de un Ejemplo de Vida
}

\section{I, with You. The Programme of Mediation among Equals, from the Perspective of an Example of Life}

\author{
David Lacuesta-Vizcaino * \\ Joan A. Traver-Martí \\ Universitat Jaume I, España
}

\begin{abstract}
Aprender a gestionar los conflictos entre las personas es vital para el desarrollo de una mentalidad cívica y de una convivencia pacífica. Los centros educativos están repletos de conflictos entre los individuos que allí confluyen y, algunos de ellos, optan por implantar nuevos métodos de resolución de conflictos, pasando del castigo y la sumisión al diálogo y la reflexión del alumnado, como lo es el programa de mediación entre iguales. El objetivo de esta investigación es conocer cómo se desarrolla el programa de mediación entre iguales, y cuál es la función del/la psicopedagoga en él. Para ello, en esta investigación biográfico narrativa, construiremos la historia de vida de una psicopedagoga que implantó un programa de mediación entre iguales en un centro de educación secundaria, analizando el proceso que se llevó a cabo y como se introducía la figura de la psicopedagoga dentro del mismo, teniendo en cuenta las voces de las personas involucradas en todo el proceso. Los resultados avanzan la necesidad de un equipo formado en mediación, además de una concienciación de toda la comunidad educativa, con el fin de poder asentar en el centro una cultura donde el diálogo y la igualdad sean referentes. Así pues, la psicopedagoga se convierte en una agente de cambio y renovación pedagógica, trabajando de forma colaborativa con la comunidad educativa, para mejorar la convivencia en el centro.
\end{abstract}

Descriptores: Solución de conflictos; Mediación; Biografía; Educación para la paz; Psicología de la educación.

\begin{abstract}
Learning to manage conflicts between people is vital to the development of a civic mindset and peaceful coexistence. Schools are filled with conflicts between the individuals who converge there. Some schools opt for new methods of conflict resolution, from punishment to dialogue and reflection of students, such as is the mediation among equals. The objective of this research is to know how the program of mediation among equals is developed, and what the function of the psychopedagogue in it is. For that, in this narrative biographical research, we will build the history of a psychopedagogue that implemented the programme of mediation among equals in a secondary education center, analyzing how the process went and what its function has been, taking into account the voices of the people involved in the whole process. The results advance the need for a team formed in mediation, in addition to an awareness of the entire educational community, with the purpose of being able to settle in the center a culture where the dialogue and the equality are referring. Thus, the psychopedagogue becomes an agent of pedagogical change and renewal, working collaboratively with the educational community to improve coexistence in the center.
\end{abstract}

Keywords: Conflict resolution; Mediation; Biographies; Peace education; Educational psychology.

*Contacto: al260230@uji.es

ISSN: 1696-4713

www.rinace.net/reice/

revistas.uam.es/reice
Recibido: $\quad 1$ de septiembre 2017

$1^{\text {a }}$ Evaluación: 11 de octubre 2017

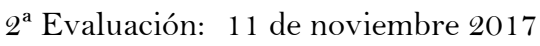

Aceptado: $\quad 5$ de diciembre 2017 


\section{Introducción}

Los seres humanos somos seres sociales y por ello hemos creado un estilo de vida al cual hemos llamado vivir en sociedad. La convivencia con otras personas lleva implícitos conflictos entre la gente, amistades, vecindario o familiares. Un centro escolar es una sociedad en miniatura y en él conviven múltiples agentes de la comunidad educativa, como profesorado, familias, alumnado y personal no docente. Cuando hablamos de conflictos entendemos que son la consecuencia de un problema, entendido este como alguna dificultad en algún aspecto organizativo, relacional o personal. Esta dificultad se transforma en un conflicto cuando enfrenta a individuos que poseen puntos de vista diferentes en relación al problema (Teixidó, 1999). La escuela, entendida como un espacio donde conviven personas, propicia que dentro de ella existan relaciones entre ellas, personas con disparidad de opiniones, intereses o motivaciones (Lúcio y Neves, 2010).

La escuela debe convertirse en un lugar de aprendizaje en cuanto a gestión de conflictos se refiere. El número de situaciones conflictivas que allí suceden nos ofrecen la oportunidad para aprender a gestionar de forma positiva los problemas de convivencia (Luna, 2012). Además, algunos autores remarcan que las herramientas que se les proporcionen al alumnado deben estar "adaptadas a las nuevas dinámicas de convivencia actuales que exigen, no sólo obtener los objetivos deseados, sino también mantener las relaciones interpersonales como base de una convivencia pacífica, no violenta y respetuosa con los demás" (De Prada y López, 2008). Así, en la mediación entre iguales encontramos una herramienta en la cual se priman las relaciones, el diálogo y la posibilidad de encontrar una solución que sea adecuada para ambas partes del conflicto. Para llevar a cabo el programa de mediación entre iguales, podemos contar con la figura del psicopedagogo o la psicopedagoga mediadora, quien se encarga de coordinar el proceso y formar tanto a profesorado como alumnado. Además, este programa lo puede abordar desde sus funciones legislativas, a partir de sus funciones más pedagógicas.

En este artículo pretendemos conocer cómo se desarrolló, desde el trabajo de la psicopedagoga, el programa de mediación entre iguales en un centro donde obtuvo resultados positivos. Para ello, hemos realizado una Investigación Biográfico Narrativa (IBN) en la que nos aproximamos a la historia de vida de la psicopedagoga que llevó a cabo dicho programa. Como podremos apreciar, la buena implantación del programa depende del grado de implicación del centro con el cambio, y precisa además de un equipo formado en mediación, entre los cuales, se incluye a la psicopedagoga, quien en este caso puso en marcha dicho proceso. Como conclusión, los participantes de esta investigación resaltan la necesidad del apoyo del equipo directivo de los centros, además de un compromiso de toda la comunidad, incluyendo al personal no docente y familias.

\section{Fundamentación teórica}

Es bueno entender los conflictos como una oportunidad para la mejora tanto personal como a nivel colectivo y comprender que son un aspecto inevitable en nuestra sociedad (Horowitz, 1998). Pero conflictos existen de muchos tipos. Tal como recoge Teixidó (1999), los conflictos más populares en un centro educativo están relacionados con las relaciones personales y afectivas. Cuando los estudios hablan de conflictos en relaciones personales, todos hacen referencia a la relación entre el alumnado, entre profesorado y 
alumnado, familia y profesorado o a nivel extraescolar, es decir, entre el centro e instituciones.

Los conflictos se pueden abordar a partir de dos modelos: a través de una mentalidad de obediencia e imposición, o a través del desarrollo de una convivencia democrática donde prima el consenso y la capacidad colectiva para la formulación de soluciones (Plasencia, 2009) y donde haya una evolución de métodos de violencia a modelos de diálogo (Oña y García, 2016). Dentro del modelo de convivencia democrática, encontramos una herramienta muy popularizada en los últimos años: la mediación entre iguales.

Cuando nos referimos al concepto de mediación, nos centramos en la acepción que explica la RAE cuando señala que mediar es "actuar entre dos partes para ponerlas de acuerdo en un conflicto”. La mediación entre iguales es un método que se empezó a utilizar unos siglos atrás en el ámbito del derecho y la economía (Miranzo, 2010), y siempre ha tenido gran peso en el ámbito jurídico por lo que se refiere a divorcios, herencias, empresas, etc. Fue a finales del siglo veinte cuando empezó a introducirse en el ámbito educativo. Se implantó en varios sistemas educativos, aunque la figura del mediador todavía no era conocida en España hasta hace apenas 20 años (Luna, 2012; San Martín, 2003). Es un hecho novedoso para la resolución de conflictos que cada vez está más extendido. Los programas de mediación entre iguales llegan a España en el año 1993, donde “aparecieron los primeros programas en el País Vasco, y pocos años más tarde los empezó a implantar la Generalitat de Catalunya” (Sánchez, 2013, p. 26). En la Comunidad Valenciana el proceso fue más tardío, ya que no llegó hasta principios del siglo veintiuno.

Actualmente, la mediación en el sistema educativo se trabaja desde tres perspectivas. Munné y Mac-Cragh, (2006) y De Prada y López, (2008) las recogen: La primera perspectiva proviene del mundo de la economía y se trata de la Escuela Tradicional-Lineal de Harvard, donde encontramos como principales referentes a Fisher o Antonio Vidal. "Su principal objetivo es que las partes lleguen a un acuerdo. Sobre todo, presta atención a la comunicación en sus aspectos verbales y entendidos de forma lineal pero no a los aspectos relacionales entre los protagonistas" (Viana, 2011, p. 73).

La segunda, llamada Circular-Narrativa, con Sara Cobb o Suares (Munuera, 2007) como principales referentes, proviene del mundo de la psicología. Esta perspectiva comprende la comunicación como un aspecto relevante en el proceso de llegar a un acuerdo final. Además, parte de las ideas del constructivismo, buscando tanto la comprensión de las relaciones que existen en el contexto como alcanzar los acuerdos, fin último de la mediación. Por último, el modelo Escuela transformativa, basado en planteamientos críticos del ámbito educativo. Entre sus máximos referentes encontramos a Folguer o Sergi Farré. Tal como explican Munné y Mac-Cragh (2006) en este modelo se pretende transformar el conflicto trabajando las diferencias entre las partes y comprendiendo el conflicto como una oportunidad de mejora.

A lo largo de los años se han ido introduciendo en las escuelas los programas de mediación entre iguales (Merino, 2005; Torrego, 2013; Villanueva, Usó, y Serrano, 2013). Todos ellos realzan la necesidad de aprender a gestionar los conflictos a partir de iguales. La manera de resolver el conflicto que entiende la mediación, ofrece una posibilidad de encontrar otros valores en torno al conflicto, ensalzando el diálogo y la mentalidad de que ganan las dos partes. Ambas deben construir la solución, buscándola de forma compartida. Se acaba produciendo una relación democrática, basada en los principios de la igualdad, olvidando aquella idea de dominio y sumisión que ha imperado durante mucho tiempo, y 
todavía sigue activa (Díaz, 2004). Por esto, los programas de mediación entre iguales buscan que sean los propios iguales quienes medien en sus conflictos, fomentando su responsabilidad, sentido social y sus habilidades para mejorar las relaciones sociales de su vida cotidiana (San Martín, 2003), fomentando la creación de una cultura de paz (Carneros y Murillo, 2017).

Desde el punto de vista de la psicopedagogía escolar, son escasos los estudios que relacionan al/la psicopedagoga como mediadora directa en conflictos, ya que casi todos los que hablan de este tema (Fernández, 2005; Selvini, 1986; Vallejos y Rodríguez, 2000; etc.) insisten en la perspectiva del/a psicopedagogo/a orientador/a y experto/a. Estos estudios explican que debe ser el orientador/a o psicopedagogo/a quien se debe encargar de las dificultades de aprendizaje, como remarca Selvini (1986),

La presencia del psicólogo/psicopedagogo en la escuela es un hecho corriente. Pero
respecto a su labor oscilan varias posturas: por un lado, afirman que es como un
asistente social común que, al igual que los demás tiene que promover la madurez
individual, social y cultural. Por otro lado, la visión del psicopedagogo como clínico
especializado que solo reconoce como ámbito operativo propio el de la intervención
terapéutica en casos de patología mental. (p. 21)

Hay autores que explican que en muchos centros puede llegar a ser considerado un extraño (Vallejos y Rodríguez, 2000). Actualmente, la figura del psicopedagogo ya se ha consolidado dentro de los centros educativos, pero su labor continúa siendo bastante clínica. Orcasitas (2002) también hace hincapié en el concepto que el psicopedagogo/a debe acompañar y ser un facilitador/a y asesor/a del claustro y de la institución educativa, a fin de mejorar, en todos los aspectos posibles, el contexto en el cual está trabajando.

Según Teixidó (1999) la clave para el logro de la gestión positiva de los conflictos reside en las personas, en los protagonistas del lugar, y el psicopedagogo, para San Martín (2003; p.159) "debe convertirse en un protagonista del contexto con un papel importante". Bisquerra (2002) resalta que, como elemento protagonista de los centros, debe convertirse en un agente de cambio de estos, donde cabe destacar características esenciales para esta investigación como son encontrar vías de resolución de conflictos desde una mirada participativa y colaborativa asegurándose encontrar la solución óptima y la capacidad de asumir un liderazgo que esté aceptado en el entorno, ya que será necesario para dinamizar los procesos de cambio.

\section{Método}

Esta investigación se enmarca dentro de un enfoque cualitativo, que pretende obtener datos y resultados a partir de las palabras de las personas (Álvarez, 2017). Dentro de este, nos centramos en la Investigación Biográfica Narrativa (Bisquerra, 2009; Bolívar, Domingo y Fernández, 2001; Moriña, 2016; Pujadas, 2002). Este tipo de investigación está basada en los presupuestos de la investigación interpretativa y comparte algunos de los principios metodológicos generales de la investigación cualitativa, sobre todo su visión hermenéutica, basada en la interpretación de textos. La IBN nos permite realizar un recorrido por la vida personal y profesional de alguien que puede ser relevante para el mundo de la educación, en nuestro caso, una psicopedagoga que impulsó el programa de mediación entre iguales en el centro donde desempeñó su función.

La bibliografía científica explica cuáles son los distintos instrumentos utilizados en este tipo de investigaciones, donde encontramos dos principales: la historia de vida y el relato 
de vida. Su intención es la misma, reconstruir una vida, pero siguiendo pautas distintas. Mientras que en el relato se presenta la historia literalmente como el narrador la cuenta, en las historias de vida el investigador ofrece una estructura intencionada a la historia. No es un relato objetivo, sino que se pretende contemplar la visión de la persona tal y como la ha vivido ella. Una historia de vida se compone de una serie de sucesos, relacionados con el fin último de la investigación (Bolívar, Domingo y Fernández, 2001). En nuestro estudio hemos optado por construir una historia de vida con polifonía de voces (Moriña, Perera, Molina, 2012) donde la visión de los informantes clave entrevistados complementa la historia de la psicopedagoga analizada.

Esta metodología cuenta con una serie de elementos que la caracterizan (Bolívar, 2012):

- Un narrador, que nos cuenta sus experiencias de vida.

- Un investigador, que interpela, colabora y "lee" estos relatos para elaborar un informe.

- Los textos, que recogen tanto lo que se ha narrado en el campo, como el informe posterior elaborado.

- Lectores, que van a leer las versiones publicadas de la investigación narrativa.

El fin último de este enfoque metodológico es la construcción de textos, los cuales nos presentan relatos biográficos a interpretar por los lectores, a los que les ofrecen una experiencia de vida, en nuestro caso de desarrollo profesional como psicopedagoga, con el que contrastar la propia experiencia y del que aprender de manera vicaria.

\section{Diseño de la investigación}

Este trabajo pretende dar respuesta a las siguientes preguntas de investigación:

- En la implantación del programa de Mediación entre iguales, tomando en cuenta la experiencia de desarrollo profesional analizada ¿cuál debe ser la función del psicopedagogo/a?

- En función de la información recogida relativa a la experiencia de desarrollo profesional analizada, ¿A qué factores negativos (problemas) y positivos (facilitadores) se enfrenta el/la psicopedagoga durante el proceso de implantar el programa de mediación entre iguales?

- ¿Cuál ha sido el desarrollo profesional de esta psicopedagoga antes y después de implantar el programa de mediación entre iguales?

Para llevar a cabo este trabajo hemos seguido las fases que normalmente se plantean en la IBN (Álvarez, 2017) y para dar cuenta del trabajo realizado las hemos ordenado de manera cronológica: Revisión de la literatura, selección de la muestra, acceso al campo de investigación, técnicas de recogida de información, análisis de los datos y construcción de la historia de vida.

\section{Revisión de literatura}

En primer lugar, realizamos un proceso de revisión de la literatura especializada, en repositorios de varias universidades del panorama nacional, publicaciones en revistas relacionadas con la temática investigada, las bases de datos Dialnet, Mendeley, TDX, academia.edu y en bases de datos internacionales como ERIC, Latindex o el buscador de Google Académico. El proceso ha sido constante durante toda la investigación. 


\section{Selección y construcción de la muestra}

Para seleccionar la muestra se realizó un muestreo intencional. Esta muestra está constituida por una única persona, la psicopedagoga de un centro de educación secundaria. Ella es quien desencadena todo el proceso de la implantación del programa de mediación entre iguales en su instituto, y el interés de la investigación era encontrar y relatar la historia de un/a psicopedagoga que hiciera mediación o estuviese implicada en procesos semejantes. El programa resultó tan exitoso, que ahora el instituto es un referente en este tema, y ella, junto con otros miembros del equipo de mediación sigue impartiendo cursos y ponencias sobre el tema, como podemos observar en la siguiente nota de prensa: http://www.radiovila-real.es/el-ies-miralcamp-de-vila-real-exportara-su-mediacionescolar-al-resto-de-centros-educativos/

\section{Acceso al campo de investigación}

Tal y como recomienda Simons (2011), en primer lugar, debemos crear un clima de confianza entre las partes -protagonista e investigador- y entrar en su universo de la forma menos agresiva posible.

En nuestro caso, primero contactamos con el actual psicopedagogo del instituto donde la protagonista se jubiló. A través de él, la psicopedagoga objeto de estudio conoció esta investigación y aceptó participar, fecha en que se inician nuestras conversaciones telefónicas, siempre orientadas a quedar presencialmente, pues la distancia entre las ondas no acaba de crear el clima humano necesario para conocernos más. En la primera reunión presencial, celebrada en una cafetería de la ciudad, conversamos, de manera informal, acerca de su trayectoria. La protagonista agradeció que estuviera informado de sus logros y algunos aspectos de su trayectoria profesional, lo cual, permitió establecer un espacio de mayor confianza entre nosotros. También en esta reunión, le fue entregado el documento del "consentimiento informado". En él se detallaba la confidencialidad de los datos, qué instrumentos se utilizarían para la recogida y análisis de la información, y cuáles eran los derechos de la protagonista a lo largo de la investigación, agradeciendo, finalmente, su colaboración.

\section{Informantes claves}

Durante el transcurso de las entrevistas, la protagonista mencionó algunas personas que la habían acompañado a lo largo del camino, y que, para ella, podían ser muy útiles para la investigación. Los datos aportados por estos informantes se utilizaron para triangular la información, y para que aportaran también su visión externa en relación con la tarea de la psicopedagoga. Asimismo, la polifonía a partir de la que se construye la historia se realiza utilizando la voz de estos informantes.

\section{Selección de los escenarios}

Dado el buen clima que se creó entre protagonista e investigador, la psicopedagoga propuso el salón de su casa para la realización de las entrevistas: un lugar cómodo, sin ruidos, íntimo. Comodidad para la grabación, y comodidad para ella, quién en un ambiente más cálido y familiar, estaría más dispuesta a abrirnos las puertas de su vida. 


\section{Técnicas de recogida de información}

Para llevar a cabo la investigación, utilizamos diversas técnicas: la entrevista biográfica, el diario del investigador y el análisis documental.

De acuerdo con Taylor y Bogdan (1987) la entrevista es un encuentro cara a cara entre el investigador y el informante con el fin de comprender las perspectivas, a través de las palabras. En esta investigación se ha utilizado la modalidad de entrevista biográfica. La entrevista biográfica es la modalidad más común dentro de la IBN. Se trata de un diálogo poco pautado, con una estructura abierta y flexible (Pujadas, 2002) donde se pretende inducir a los protagonistas a que reconstruyan su vida, siguiendo un orden lógico y con respuestas claras. La entrevista elaborada para este caso sigue los esquemas de Bolívar, Domingo y Fernández (2001) y se ha dividido en los cuatro tiempos que proponen:

- Esbozo biográfico general (breve resumen de su historia de vida).

- Primera etapa: Primaria y bachillerato.

- Segunda etapa: Estudios y año de carrera.

- Tercera etapa: Ejercicio profesional.

Con la intención de clarificar la información que había quedado diluida y profundizar con más detalle en las preguntas de la investigación, se realizó una entrevista focalizada sobre el proceso de la implantación del programa de mediación entre iguales, centrando toda su atención en aquellos aspectos realmente importantes para esta. Finalmente, para triangular la información recogemos la voz de los que han acompañado a nuestra protagonista a lo largo de su carrera. Se realizaron entrevistas a otros informantes, los cuales han estado presentes en la vida profesional y personal de la protagonista. Estas complementan las anteriores, y se consideran entrevistas semiestructuradas (Moriña, 2016).

El diario de campo tiene como finalidad registrar aquellas observaciones que el investigador pueda considerar relevantes para la investigación (Martínez, 2007) y que posteriormente facilitaran la comprensión de los datos finales. Por último, el análisis documental tiene como una de sus finalidades conocer segmentos específicos de información de algún documento en particular (Peña y Pirela, 2007), el cual es utilizado en este trabajo para poder analizar la legislación vigente en torno a la figura del/la psicopedagoga.

Además, a cada instrumento y participante se le ha asignado un código: Entrevista Biográfica Psicopedagoga (EB), Entrevista Focalizada Psicopedagoga (EF), Entrevista Exdirector (EEX), Entrevista Psicopedagogo (EPS), Entrevista Profesor (EP), Análisis audiovisual (AA) y Análisis documental (AD)

\section{Análisis de datos}

En esta investigación, el análisis de datos se ha realizado durante el proceso de recogida de información. Tras la realización de las entrevistas, se llevó a cabo su transcripción de forma literal, para así poder trabajar más cómodamente con la información.

Con la información registrada se han realizado dos tipos de análisis: narrativo y estructural. Con el objetivo de elaborar la historia de vida, en el análisis narrativo (Bolívar, Domingo y Fernández, 2001; Moriña, 2016), se realizó una lectura exhaustiva de todas las transcripciones. A través de ella se encontraron momentos significativos que 
explicaban la trayectoria personal y profesional de la protagonista, centrándose la parte final en la implantación del programa de mediación entre iguales. Estos momentos los identificamos como incidentes críticos ya que, como explica la psicopedagoga entrevistada, suponen situaciones que han influido de manera importante en su desarrollo profesional.

En el análisis estructural (Bolívar, Domingo y Fernández, 2001; Moriña, 2016), se ha realizado un análisis de categorías, a partir de una reducción de datos en función de las categorías extraídas previamente. Estas son del tipo deductivo: la visión del conflicto, cultura mediadora; e inductiva como formación previa, barreras, el apoyo del equipo directivo o el seguimiento del programa. Una vez localizadas las categorías que ayudarán a responder nuestras preguntas, podemos establecer relaciones entre ellas. A través de la triangulación de la información recogida podemos otorgarles validez a estas.

Construcción de la historia de vida

La historia de vida de esta investigación se ha construido a partir de incidentes críticos. Se ha buscado una frase desencadenante y se ha construido el relato a partir de la reducción de información asociada a cada incidente crítico.

Mediante un lenguaje narrativo, se ha hecho un relato en primera persona, siguiendo un hilo que ha ido pasando por los incidentes críticos, a partir de una visión cronológica, de lo más antiguo hasta el presente. En la historia de vida aparecen tanto la voz de la psicopedagoga como la visión de los informantes clave que refuerza en ciertos momentos el transcurso de la historia, triangula la información recogida y convierte el relato final en una historia de vida polifónica.

Una vez elaborada, la historia fue retornada a su protagonista, quien puntualizó, eliminó y añadió información, de tal forma que se llevó a cabo una co-construcción del relato (Álvarez, 2017; Moriña, 2016).

\section{Resultados}

Este trabajo presenta dos resultados principales: el primero, el análisis estructural, nos permite responder a las dos primeras preguntas de la investigación; el segundo, fruto del análisis narrativo, da lugar a la historia de vida.

\subsection{Análisis estructural}

A continuación, vamos a tratar de responder a las preguntas de investigación a través de la información recogida en las entrevistas, del análisis audiovisual y del análisis de documentos escritos, realizando un análisis estructural de la información. Previamente hemos elaborado un mapa en el cual se aprecia la relación entre las diferentes categorías de análisis.

En la implantación del programa de Mediación entre iguales, ¿cuál debe ser la función del psicopedagogo/a?

En primer lugar, observamos cuales son las tareas psicopedagógicas enmarcadas en la legislación y de qué forma las atiende la psicopedagoga, desde su visión y el análisis de la normativa (figura 1). En segundo lugar, observaremos cómo podemos incluir la implantación del programa de mediación entre iguales (figura 2). 


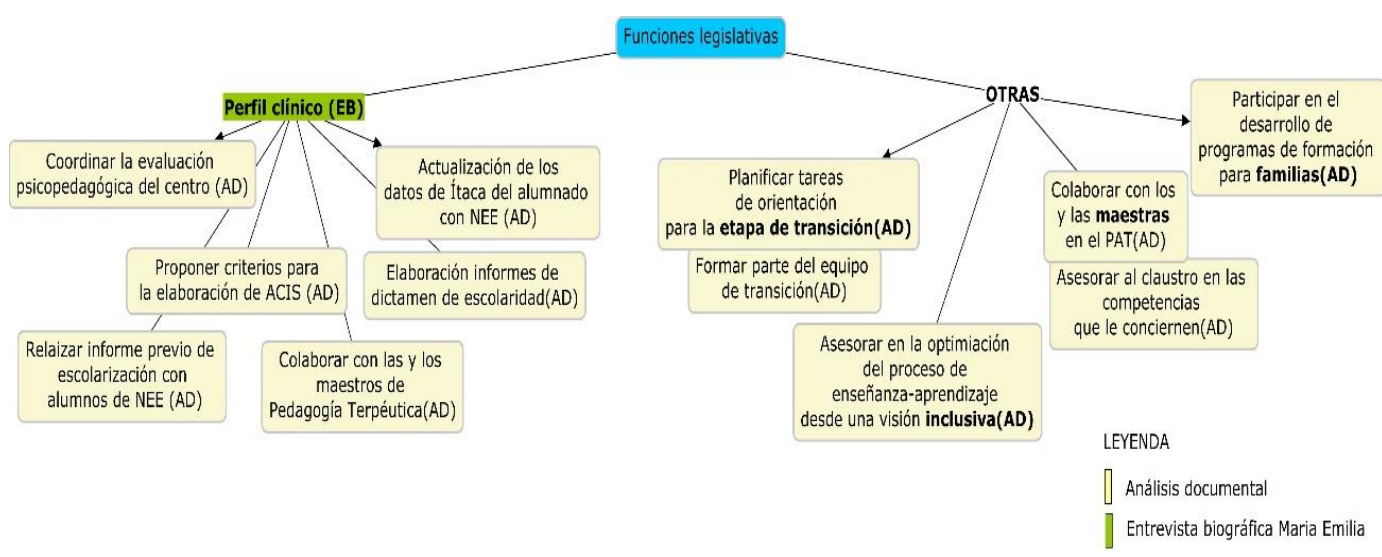

Figura 1. Pregunta investigación $n^{\circ}$ 1, primera parte

Fuente: Elaboración propia.

Según la legislación vigente en la Comunidad Valenciana (orden 7826 del 12 de Julio de 2016), todavía no está claro todo el abanico de tareas que pueden realizar los profesionales de la psicopedagogía en los centros educativos, oscilando entre posturas más clínicas y otras que versan entre asesorar en procesos de formación tanto a familias, claustro y/o profesores (AD). Para la psicopedagoga predominan las tareas de índole clínica, siendo estas la mitad de sus funciones, y dejando las otras seis funciones a temas como las familias, claustro, etapas de transición y un solo artículo con referencia a postulados inclusivos. En relación a este hecho, la psicopedagoga se ve "más pedagoga que psicopedagoga, iba más a programas grandes, que a atenciones de tipo terapéuticas” (EF).

En el artículo 3.1. (k) de la orden referenciada, se explicita que una de las tareas del/la psicopedagoga es asesorar al claustro en aspectos requeridos en el ámbito de sus competencias (AD). La gestión de la convivencia del centro forma parte de sus competencias y, en este centro, se decidió gestionar los problemas de convivencia a través del programa de mediación entre iguales.

Del trabajo analizado de la experiencia profesional sobre la psicopedagoga protagonista en este trabajo, se extraen una serie de factores que, tanto previa como posteriormente han influido en el éxito del programa de mediación entre iguales

En el momento de iniciarlo y durante todo el proceso, se entiende que tiene que ser un proceso democrático, igual que lo es la mentalidad mediadora, donde se necesita, en primer lugar, de unos valores: "la persona no es solo inteligencia, ni solo habilidades sociales, ni solo emociones. Debe ir todo a una, porque si en mediación yo no tengo claro cuáles son los valores mínimos, puede que mi solución sea dañina para la otra persona" (EF).

Uno de estos valores que ofrece la mediación entre iguales es la resolución del conflicto a través del diálogo, ya que "con la mediación entre iguales proporcionamos una herramienta que pasa del camino de la agresión y sumisión al del diálogo, lo cual luego es aplicable al día a día para mejorar la convivencia” (EF). Así pues, la resolución de conflictos a través de la mediación entre iguales persigue que "las partes implicadas lleguen a un acuerdo por sí solas” (AA). 


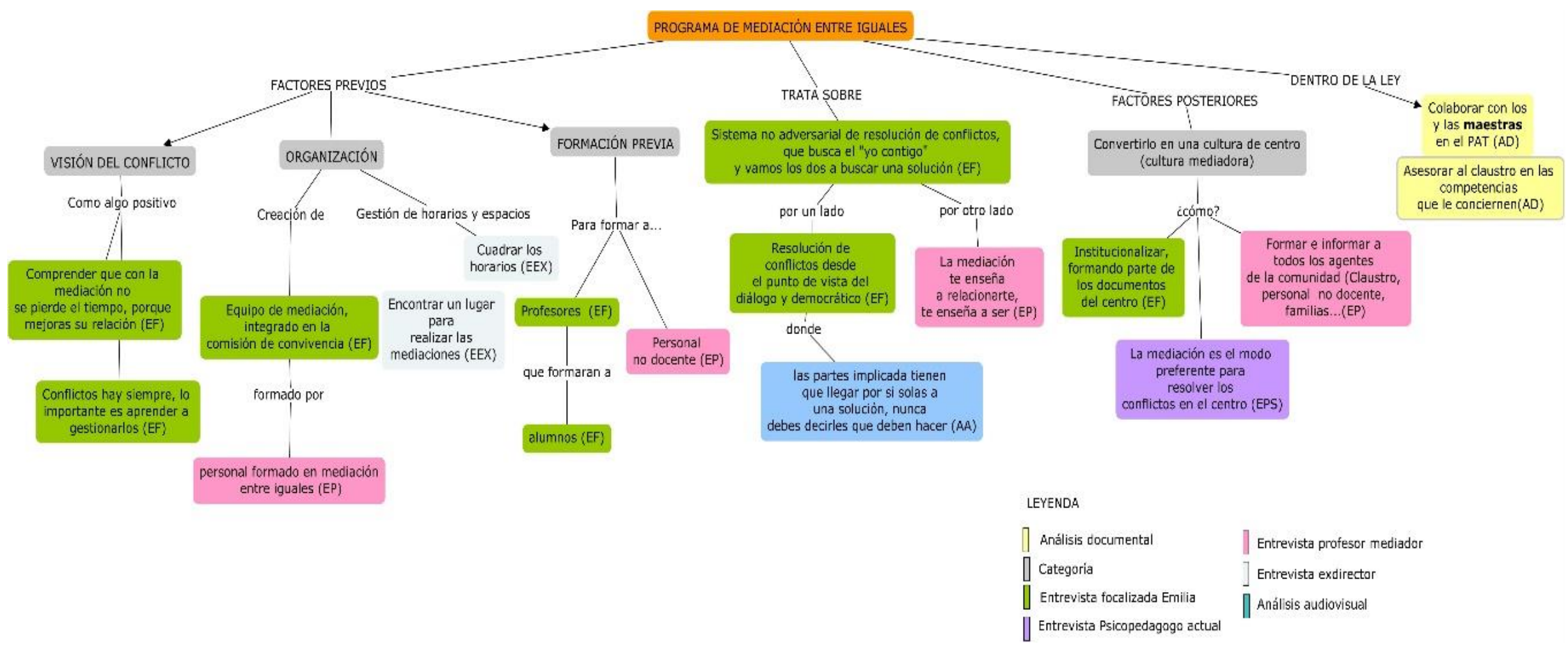

Figura 2. Pregunta investigación $n^{\circ}$ 1, segunda parte Fuente: Elaboración propia. 
Para poder desarrollarlo con éxito se requieren una serie de factores previos. Lo más esencial en la implantación del programa de mediación entre iguales es cambiar la visión sobre lo que significa el conflicto y como éste se puede gestionar. El conflicto tiene que entenderse como una oportunidad positiva, presente "en todas las caras de la vida, por eso es importante aprender a gestionarlos" (EF).

Además, desde el punto de vista de la psicopedagoga, es importante la existencia de una buena organización de todo el sistema del programa, empezando por la creación de un organismo controlador: "se tiene que configurar un equipo de mediación, con formación previa en el tema, que luego tirarán del carro” (EF). El psicopedagogo/a puede ser quien "coordine" (EP) el grupo, y no ocuparse de todas las tareas. Para esto, es necesario formación previa, donde se enseñe a los "profesores" (EF) y que estos posteriormente formen al alumnado. Uno de los pilares del programa reside en cómo se gestionan los horarios y los espacios, y eso no puede hacerlo la psicopedagoga sola, sin la colaboración de figuras importantes como el equipo directivo: "La psicopedagoga lo impulsó con la ayuda de la jefa de estudios, que cuadró todos los horarios" (EP); "Yo delegué en ellas, mientras que seguía con la dirección, y la jefa de estudios y la psicopedagoga lo pusieron todo en marcha" (EEX).

También existen factores posteriores que afectan a la consolidación del programa, donde el objetivo primordial es convertirlo en cultura de centro. Por esto, la mentalidad que perseguía el grupo era "que los padres y madres también estuvieran formados o, al menos, informados; tener al personal no docente también formado, como Pepe el conserje, que lo está. Lo ideal sería que toda la estructura del centro estuviera formada" (EP) y "sensibilizar a toda la comunidad, implicándolos, a todos los que intervienen en el centro" (EF). La psicopedagoga, se convierte en lo que algunos compañeros llaman "psicopedagoga mediadora" (EP) la cual "coordina, forma o acompaña en las mediaciones colaborando con la jefa de estudios y el equipo de convivencia” (EP).

Con la comunidad a favor, para que la mediación se consolide es necesaria su institucionalización, "formando parte de los documentos del centro (PEC, PAT, RRI...)" $(\mathrm{EF})$, de tal forma que consigues que "la mediación forme parte de la vida del centro y sea un sistema preferente de atención" (EPS), que trata de mejorar las relaciones entre las personas.

Cuando se inicia un programa de estas características, los protagonistas se encuentran con una serie de aspectos que dificultan su implantación y otros que la impulsan. En este caso, el programa encontró una serie de barreras que se pueden clasificar en dos tipos.

En primer lugar, la mentalidad de los agentes educativos. Frente al conflicto lo más cómodo es el castigo, tanto, que "hay gente que no se cree los procesos de mediación y prefieren medidas de tipo más punitivo" (EPS). Esto sucede por la posición que ocupan los docentes en la jerarquía de la clase, ya que "existen profesores que piensan que, si le quitas el poder de la sanción, le quitas poder" (EF) y tienen otra visión de la educación, "donde la letra con sangre entra y para ellos, hablar con el alumno, es rebajarse" (EP). Algunos incluso llegan a afirmar que "los alumnos son incapaces de solucionar entre ellos sus problemas" (EF). 


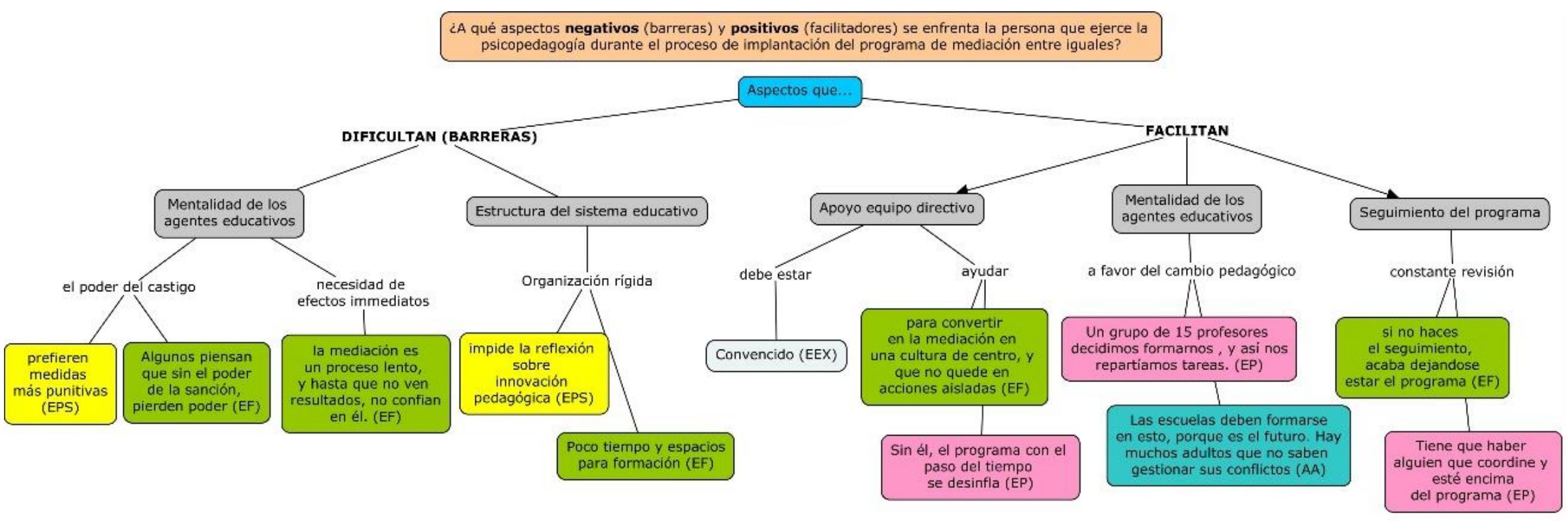

LEYENDA

$\begin{array}{llll}\square & \text { Categoría } & \square & \text { Entrevista profesor mediador } \\ \square & \text { Entrevista focalizada Emilia } & \square & \text { Entrevista exdirector } \\ \square & \text { Entrevista Psicopedagogo } & \square & \text { Análisis audiovisual }\end{array}$

Figura 3. Pregunta de investigación $n^{\circ} 2$ Fuente Elaboración propia. 
Para implantar el programa, el exdirector cuenta como la psicopedagoga, tuvo que realizar mucho trabajo por detrás, porque "había que cuadrar muy bien los horarios, molestando lo menos posible y convenciendo lo más" (EEX) y junto con la jefa de estudios, "había que facilitarles las cosas a los profesores, ya que a la gente las cosas nuevas les dan...” (EEX).

La mediación entre iguales exige un proceso lento, entre la formación y la asimilación de ésta como recurso eficaz, y esto también creaba reticencias entre algunos miembros del claustro. "La sociedad en la que estamos solo quiere resultados inmediatos, es incapaz de esperar" (EF) y esto se refleja en la educación, pero cuando se ven resultados, la gente empieza a reaccionar: "los que al principio ponían trabas a la mediación, con el tiempo vieron su eficacia y, aunque no se implicaron, dejaron de poner dificultades" (EP), y "hasta que los profesores no lo asumen, lo ven como una pérdida de tiempo" (EF).

La segunda barrera tiene que ver con cómo se estructura el sistema educativo, ya que el tiempo laboral es fundamental para poder implantar programas de gran impacto en el centro, y "algunos profesores nos reñían si sacábamos al alumnado de su aula, durante la clase, que era cuando se podían hacer las mediaciones” (EF). Además, esta organización tan rígida y cuadriculada impide la reflexión: "en los institutos en estos momentos no tenemos espacios ni tiempos para dedicar al debate pedagógico y por tanto no hay diagnósticos compartidos y muchas cosas se hacen sin reflexionar" (EPS).

En cuanto a los aspectos positivos, en primer lugar, la necesidad del apoyo del equipo directivo, porque "en un centro donde el equipo directivo no está por la labor, con el tiempo se desinfla" (EP), "el equipo directivo debe estar convencido, y yo las ayudaba en todo lo que podía porque han demostrado que es realmente útil" (EEX) terminando con que la psicopedagoga explica que "sin el apoyo del equipo directivo, se queda en acciones aisladas, y eso no es lo que busca la mediación, la mediación es una cultura” (EF).

La mentalidad de los agentes educativos también se convierte en un punto fuerte, ya que aquellos que abogan por el cambio pedagógico, se implican en el programa. En este caso fue un grupo de 15 profesores que, tras la formación recibida por la psicopedagoga, decidieron ponerlo en marcha: "decidimos formarnos y al terminar configuramos un grupo donde nos repartíamos tareas" (EP). Las familias, al conocerlo, explican que "esto es el futuro, y las escuelas deben formarse en esto" alegando que "hay muchos adultos que no saben gestionar sus conflictos" (AA). Por su parte, el alumnado mediador ha entendido los valores democráticos que existen tras la mediación: "no juzgamos, solo dialogamos", "te hace pensar que en las relaciones entre personas siempre hay cosas que no ves” (AA).

Por último, es necesario realizar un "seguimiento constantemente", "Si no le haces seguimiento, el programa se acaba diluyendo" (EF). La propia psicopedagoga explica que existen centros que lo intentan implantar, pero fracasan por no estar encima, debido a que "si decides poner un programa en marcha, pero luego nadie realiza el seguimiento, acaba perdiendo fuerza" (EF).

\subsection{Análisis narrativo}

$\mathrm{Al}$ atender los requerimientos de la publicación no hemos podido incluir toda la historia de vida debido a la limitación del espacio. Para dar cuenta de la misma y presentar este resultado, seguidamente ofrecemos el índice del relato y un extracto de la historia de vida. A continuación, y para finalizar este apartado presentamos un extracto de la historia de vida que ilustra cómo se ha elaborado su narrativa: 
Miralcamp no era un centro conflictivo, era un centro como otro. Te he explicado que estaba situado en las afueras de Villarreal, y tanto la distancia como la Formación Profesional-que no era valorada en aquel tiempo-, lo hacían un centro desconocido y considerado de segunda categoría para muchos ciudadanos. El equipo del centro creímos que la fórmula para erradicar los conflictos que salieron en el centro era "el expediente disciplinario". Estrictos, pasaban muchas cosas alli y nosotros no pasábamos ni una. Íbamos a muerte. Creíamos que así se solucionaban las cosas. El alumno, una vez ha cumplido la sanción, su responsabilidad ha terminado. $\Upsilon$ tú no consigues modificar la conducta, simplemente sancionarla. $Y$ llegó el curso de hacer 35 expedientes disciplinarios.

Dedicaban horas y horas a realizar expedientes disciplinarios. Días, donde el director y jefe de estudios se habian dedicado unicamente a hacer expedientes. ¿̇Sabes qué le supone de tiempo al equipo directivo hacer un expediente? Vale la pena probar programas experimentales. De la otra forma no conseguimos nada, sólo que el centro se convirtiera en enemigo del alumno, y cuando algo es enemigo tiendes a joder todo lo que puedes. Esto quien volvía, porque algunos de ellos, al segundo expediente ya no volvían y provocaba abandono.

Insatisfechos con la forma de gestionar la convivencia, decidimos revertirla. ¿Cómo? Innovando, buscando alternativas para gestionar la convivencia, buscando cambiar la mentalidad y pasar de "la acción-reacción" a "la acción-educación". Nos encontramos con un método de resolución de conflictos: la mediación entre iguales.

Decidimos hacer un curso del Cefire y empezamos la implantación del programa formándonos el claustro primero. Era yo quien formaba a la gente, al tiempo que yo también me estaba formando: leíamos, buscábamos, debatíamos. Con la ayuda de la jefa de estudios, que organizó el horario para formarnos, hicimos un grupo de formación donde estábamos dispuestos a cambiar las cosas. En el grupo repartíamos tareas, y yo me encargaba de coordinarlo. Por supuesto, contábamos con todo el apoyo del equipo directivo. [...] Al principio de empezar a hacer mediaciones, teníamos reticencias de algunos profesores que se quejaban porque sacábamos los alumnos del aula y nos acusaban de hacerles perder el tiempo. No es perder el tiempo, porque ese niño irá madurando en mentalidad mediadora y resolutiva, y te bajarán los conflictos en el aula, por lo tanto, te estamos haciendo ganar tiempo. Cuando empiezan a ver resultados todo cambia. (Extracto de la historia de vida de la psicopedagoga)

\section{Discusión y conclusiones}

Durante el proceso de revisión de literatura, nos encontramos con lo que autores como Usó, Adrián y Villanueva (2010) o San Martín (2003) comentan: a nivel científico la mediación no cuenta con abundante bibliografía. Aspecto que además se incrementa cuando intentamos averiguar cuál es el estado de la cuestión con respecto a los tres ejes que configuran la temática de trabajo de nuestra investigación: mediación entre iguales, psicopedagogía e investigación biográfica narrativa. Hecho que hemos podido constatar en nuestro proceso de revisión de la literatura científica.

Para presentar las conclusiones, se realizará a partir de las preguntas de investigación. De cada una de ellas, se han extraído una serie de conclusiones principales que posteriormente estarán relatadas.

En referencia a la primera pregunta de investigación, que hacía referencia a la función del/la psicopedagoga durante la implantación del programa de mediación, se evidencia una confusión legislativa, además de una visión clínica frente a lo pedagógico. Los documentos legislativos son muy ambiguos e interpretables, haciéndose visible únicamente su trabajo con las dificultades de aprendizaje y los trastornos. Desde que se instauró la figura del psicopedagogo/a en el año 1990 con la llegada de la Ley Orgánica 
1/1990 del 3 de octubre de Ordenación General del Sistema Educativo (LOGSE), su función ha sido confusa debido al gran abanico de tareas que puede abarcar (Bisquerra, 2002) aunque se ha ido modificando desde unas posturas más clínicas hacia unas más inclusivas, con todavía un largo camino que recorrer.

En nuestro caso, la psicopedagoga explicita que ella era pedagoga y no psicopedagoga, lo cual explica porque se decanta por programas como la mediación, más cercanos a una orientación pedagógica a nivel de centro, que a un perfil de psicología clínica. Posiblemente, el hecho de que ella se haya dedicado más a los temas de mediación y de programas de centro, es debido a que en su formación estaba la impronta de la pedagogía y su mirada iba más hacia aspectos de este tipo. Esto lo ha podido desarrollar, sobre todo, en las funciones menos arraigadas a la vertiente clínica.

En cuanto al programa de mediación entre iguales, la principal conclusión que extraemos es que logra ser exitoso cuando se convierte en "cultura del centro". Para esto se necesita implicar a toda la comunidad para la creación de una cultura de paz (Carneros y Murillo, 2017), una cultura mediadora, donde los conflictos se resuelven de forma dialógica (Luna, 2012; Oña y García, 2016), incluso, sin recurrir a una mediación formal. Para crear esta cultura de centro es importante un trabajo previo: En primer lugar, y siguiendo los estudios de Horowitz (1998), nuestra visión sobre el conflicto debe ser positiva. Entenderlos como oportunidad para mejorar las relaciones entre las personas, y no como un hecho a erradicar, nos permitirá entender estrategias como la mediación entre iguales.

Por otro lado, no es función únicamente del profesional de la psicopedagogía implantar estas iniciativas, sino que es muy importante la existencia de un equipo, implicado en formarse y desarrollar el programa. En este caso, la psicopedagoga se convierte en coordinadora de todo el grupo, sin realizar ninguna tarea concreta, sino que, al igual que sus compañeros, formaba alumnos mediadores, organizaba sesiones de formación, acompañaba en las mediaciones... Se necesita un liderazgo de tipo democrático (López et al., 2014; Murillo, 2006), ligado a los principios de la medicación entre iguales, a través del cual se potencia la participación y colaboración de todo el grupo, hecho que garantiza el mantenimiento del programa y el éxito que obtuvo. Esto implica un cambio de mentalidad, alejado de las tareas individualizadas típicas de la psicología clínica. Un/a psicopedagoga con una mentalidad clínica, lo habitual en la literatura (Fernández, 2005; Selvini, 1986; Vallejos y Rodríguez, 2000), no le permite estar presente en programas de gran centro con una dedicación total.

Teixidó (1999) explica que gestionar la convivencia es tarea de los protagonistas del contexto, y la mediación tiene que surgir como una necesidad de cambio metodológico. Mundina (2017) explica que no puede imponerse desde fuera, tiene que ser una necesidad del centro. La mediación entre iguales es solamente un planteamiento más para gestionar la convivencia. Un planteamiento que te enseña a gestionar los conflictos a partir de valores democráticos, donde nadie tiene más poder que el otro, y por lo tanto deben colaborar para llegar a una solución a través del diálogo (Boqué, 2003; De Prada, y López, 2008; Sánchez, 2013). Si aprenden una forma de enfocar los problemas hacia soluciones, les resultará muy útil para su vida cotidiana. Por esto, la mediación entre iguales implica un modelo de centro basado en la convivencia democrática (Plasencia, 2009), ya que los valores que hay detrás de la cultura de la mediación facilitan el aprendizaje de valores humanos y habilidades que permiten el desarrollo integral y cívico del alumnado. 
En relación a la segunda pregunta de investigación, referida a los factores a los que se enfrenta la psicopedagoga durante el proceso, las principales conclusiones son la necesidad del apoyo del equipo directivo como elemento facilitador, y la mentalidad de los agentes educativos como elemento distorsionador. Los resultados señalan una serie de limitaciones y facilitadores enmarcados, en el caso de la psicopedagoga, en el proceso de implantación del programa. Dentro de la categoría barreras, podemos diferenciar entre dos tipos: aquellas de carácter más personal, y otras con carácter organizacional, aunque podemos relacionarlas. Debido a la organización jerárquica de los centros educativos, los estamentos que se sitúan en la zona alta (profesorado) poseen más poder que los de la zona baja (alumnado). Esto ocasiona acomodación por parte de este sector, el cual utiliza la estrategia del castigo frente al conflicto. Impidiendo así buscar alternativas más dialógicas, donde exista una relación horizontal en la resolución del conflicto, y sean las partes quienes propongan la solución (Oña y García, 2016).

En referencia a la categoría facilitadores, destacamos antes que nada la necesidad del apoyo del centro y del equipo directivo. En el centro de nuestra protagonista sí que han contado con él, y el programa se fue extendiendo hasta crearse una cultura mediadora en el centro, gracias a la implicación y compromiso. De acuerdo con el Departamento de Justicia y Administración Pública del Gobierno Vasco (2011), el primer paso es lograr el compromiso del centro, del equipo directivo, del claustro y del órgano máximo de representación o consejo escolar.

Cabe destacar que, tanto si es el psicopedagogo/a o el profesorado quien emprende el programa de mediación, los pilares básicos en torno a él son estos, a partir de los cuales se consigue un resultado exitoso en su implantación.

Como toda investigación cualitativa, todo depende de las personas. Tal vez el éxito del programa en este caso tenga su efecto en la visión que tiene el centro educativo sobre la psicopedagoga, sobre la visibilidad y reconocimiento de su trabajo, y en relación a su mentalidad y actitud como agente de cambio y mejora escolar. Innovación y psicopedagogía son dos palabras que se deben coger de la mano, ya que su figura está presente en los centros, y no puede convertirse en un elemento sin importancia. Cuando estas dentro de la escuela, eres parte de la escuela. En tus manos está utilizar el poder que se te ha concedido. La experiencia de desarrollo profesional analizada como psicopedagoga en un centro de enseñanza secundaria en relación a los procesos de mediación escolar, nos ofrece un rico marco de trabajo con el que contrastar y del que aprender en nuestra labor educativa.

Una de las limitaciones de esta investigación tiene que ver con ciertas dificultades de acceso a la muestra desde una perspectiva longitudinal. En un principio, desde el diseño de la investigación realizada, pretendíamos acceder a un repertorio algo más amplio de informantes clave que abarcara todo el tiempo que lleva en marcha el programa de mediación en el instituto. Aunque intentamos ponernos en contacto con varios de estos referentes, por distintos motivos de agenda y disponibilidad, no se pudieron llevar algunas acciones que hubieran enriquecido la investigación, como son entrevistas a exalumnos participantes del programa de formación, o entrevistas con otros profesionales que estuvieron en contacto directo con la psicopedagoga protagonista de esta investigación.

Con la visión de la psicopedagoga ya expuesta, una dirección que podría tomar esta investigación está en descubrir las voces de los protagonistas centrales del programa de mediación entre iguales: el alumnado. Indagar sobre cuál ha sido su percepción acerca del 
programa, desde la perspectiva de alumnado mediador y desde la perspectiva de alumno que estuvo involucrado dentro de un proceso de mediación, nos ayudará a comprender cuál es su percepción acerca de todos los elementos que constituyen un programa de mediación entre iguales, como es el rol del profesorado, el centro educativo o la propia mediación.

\section{Referencias}

Álvarez, E. (2017). Narrativa de éxito educativo y social desde las voces silenciadas de universitarias con diversidad funcional auditiva (Tesis doctoral). Universidad de Sevilla, España.

Bisquerra, R. (2002). Pasado, presente y futuro de la psicopedagogía. En Z. Martínez de la Hidalga (Ed.), Definición y perspectivas profesionales de la psicopedagogía (pp. 11-32). Bilbao: Universidad de Deusto.

Bisquerra, R. (2009). Metodología de la investigación educativa. Madrid: La Muralla.

Bolívar, A. (2012). Metodología de la investigación biográfico-narrativa: Recogida y análisis de datos. Recuperado de https://www.researchgate.net/publication/282868267_Metodologia_de_la_investigacion _biografico-narrativa_Recogida_y_analisis_de_datos

Bolívar, A., Domingo, J. y Fernández, M. (2001). La investigación biográfico narrativa en educación. Madrid: La Muralla.

Boqué, M. C. (2003). Cultura de mediación y cambio social. Barcelona: Gedisa.

Carneros, S. y Murillo, F. J. (2017). Aportaciones de las escuelas alternativas a la justicia social y ambiental: Autoconcepto, autoestima y respeto. REICE. Revista Iberoamericana sobre Calidad,

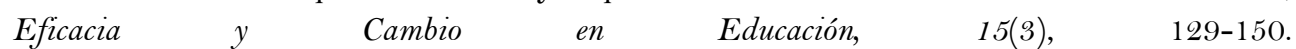
https://doi.org/10.15366/reice2017.15.3.007

De Prada, J. y López, J. A. (2008). La mediación como estrategia de resolución de conflictos en el ámbito escolar. Documentación Social, 148, 99-116.

Díaz, M. J. (2004). Prevención de la violencia y lucha contra la exclusión desde la adolescencia. Madrid: Instituto de la Juventud-Ministerio de Educación y Ciencia.

Fernández, J. D. (2005). La orientación educativa: Claves de éxito profesional. Buscando la magia del mago sin magia. Granada: Grupo Editorial Universitario.

Departamento de Justicia y Administración Pública del Gobierno Vasco. (Ed.). (2011). Cómo poner en marcha, paso a paso, un programa de mediación escolar entre compañeros. Bilbao: GEUZ.

Horowitz, S. (1998). Mediación en la escuela. Resolución de conflictos en el ámbito educativo adolescente. Buenos Aires: Editorial Aique.

López, J., García, E., Oliva, N., Moreta, B. y Bellerín, A. (2014). El liderazgo escolar a través del análisis de la actividad diaria de los directores. REICE. Revista Iberoamericana sobre Calidad, Eficacia y Cambio en Educación, 12(5), 61-78.

Lúcio, J. y Neves, T. (2010). Mediation in local educational governance: The educating cities movement in a Portuguese town. European Educational Research Journal, 9(4), 484-497 https://doi.org/10.2304/eerj.2010.9.4.484. 484-497

Luna, E. (2012). La mediación como herramienta educativa. Barcelona: Universitat de Barcelona.

Martínez, L. (2007). La observación y el diario de campo en la definición de un tema de investigación. Perfiles Libertadores, 4, 73-80. 
Merino, M. (2005). Mediación entre iguales. Monitor Educador, 108, 42-51.

Miranzo, S. (2010). Quiénes somos, a dónde vamos... origen y evolución del concepto mediación. Revista de Mediación, 3(5), 8-15.

Moriña, A. (2016). Investigar con historias de vida. Madrid: Narcea.

Moriña, A., Perera, V. H. y Molina, V. M. (junio, 2012). Sobre el uso de la historia de vida para narrar las trayectorias universitarias del alumnado con diversidad funcional. Comunicación presentada en III Jornadas de histórias de vida em educação: A construção do conhecimento a partir de histórias de vida Oporto. Oporto: Universidade do Porto.

Mundina, E. (2017). Actitud mediadora: Creando futuro. Villareal: Ediciones Intrépidas.

Munné, M. y Mac-Cragh, P. (2006). Els 10 principis de la cultura de mediació. Barcelona: Graó.

Munuera, P. (2007). El modelo circular narrativo de Sara Cobb y sus técnicas. Portularia, 7(1), 85106.

Murillo, F. J. (2006). Una dirección escolar para el cambio: Del liderazgo transformacional al liderazgo distribuido. REICE. Revista Iberoamericana sobre Calidad, Eficacia y Cambio en Educación, 4(4e), 11-24.

Oña, J. y Garcia, E. (2016). Proyecto escuela espacio de paz. Reflexiones sobre una experiencia en un centro educativo. REICE. Revista Iberoamericana sobre Calidad, Eficacia y Cambio en Educación, 14(2), 115-131. https://doi.org/10.15366/reice2016.14.2.007

Orcasitas, J. (2002). La formación del psicopedagogo/a. En Z. Martínez de la Hidalga (Ed.), Definición y perspectivas profesionales de la psicopedagogía (pp. 39-43). Bilbao: Universidad de Deusto.

Peña, T. y Pirela, J. (2007). La complejidad del análisis documental. Información, Cultura y Sociedad, $16,55-81$.

Plasencia, M. (2009). Hablar, no golpear. Razonamiento infantil sobre disciplina parental. Revista Mexicana de Investigación Educativa, 14(43), 1103-1127.

Pujadas, J. J. (2002). El método biográfico: El uso de las historias de vida en ciencias sociales. Madrid: Centro de Investigaciones Sociológicas.

San Martín, J. A. (2003). La mediación escolar. Un camino para la gestión del conflicto escolar. Madrid: Editorial CCS.

Sánchez, M. (2013). Gestión positiva de conflictos y mediación en contextos educativos. Madrid: Editorial Reus.

Selvini, M. (1986). El mago sin magia: Cómo cambiar la situación paradójica del psicólogo en la escuela. Barcelona: Paidós.

Simons, H. (2011). El estudio de caso: Teoría y práctica. Madrid: Morata.

Taylor, S. y Bogdan, R. (1987). Introducción a los métodos cualitativos de investigación. Barcelona: Paidós.

Teixidó, J. (1999). Estratègies de resolució de conflictes organitzatius en escoles $i$ instituts. Barcelona: Universitat Oberta de Catalunya.

Torrego, J. (2013). La ayuda entre iguales para mejorar la convivencia escolar. Madrid: Narcea.

Usó, I., Adrián, J. E. y Villanueva, M. L (2010). La convivencia en las aulas de secundaria. Programas alumno ayudante y alumno mediador. Castellón: Universitat Jaume I. 
Vallejos, A. y Rodríguez, R. (2000). El perfil humano y profesional del psicopedagogo desde el enfoque colaborativo de su función. Escuela Abierta, 4, 157-167.

Viana, M. I. (2011). La mediación en el ámbito educativo en España. Estudio comparado entre comunidades autónomas. Valencia: Universidad de Valencia.

Villanueva, L., Usó, I. y Serrano, J. (2013). Los programas de mediación entre iguales: Una herramienta eficaz para la convivencia escolar. Apuntes de Psicología, 31(2), 165-171.

\section{Breve CV de los autores}

\section{David Lacuesta-Vizcaino}

Graduado en Maestro de Educación Primaria, con mención en Educación Musical por la Universidad Jaume I y Máster Universitario en Psicopedagogía, también por la Universidad Jaume I de Castellón. Interesado en el campo de la Educación emocional, ha realizado investigaciones sobre la relación entre la inteligencia emocional y su vínculo con la educación musical en las escuelas de primaria. Actualmente, sus líneas de investigación versan sobre la participación ciudadana en contextos sociales, así como favorecer la educación inclusiva y educación para la paz. ORCID ID: 0000-0001-9047-9518. Email: al260230@uji.es

\section{Joan A. Traver-Martí}

Doctor en Educación, Licenciado en Filosofía y CCEE, y profesor titular del Departamento de Educación de la Universidad Jaume I de Castellón. Miembro del grupo de investigación "Mejora Educativa y Ciudadanía Crítica (MEICRI)" de la Universidad Jaume I (http://meicri.uji.es) y del "Laboratoire International sur L'Inclusion Scolaire" (LISIS) (http://www.lisis.org). Ha realizado trabajos de investigación sobre el aprendizaje cooperativo, la enseñanza de valores y actitudes y la educación intercultural e inclusiva. Tiene publicaciones y comunicaciones sobre estas temáticas. Sus líneas de investigación actuales son la educación en valores y actitudes, el aprendizaje cooperativo, la escuela intercultural e inclusiva y la participación y desarrollo comunitario. ORCID ID: 0000-0002-5948-1035. Email: jtraver@uji.es 